\title{
Improved Microanalysis Using Cathodoluminescence Spectrum Imaging with Higher Spatial Sampling
}

\author{
David Stowe, Jonathan Lee, Michael Bertilson, Salvatore Fahey and John Hunt
}

Gatan, Inc., Pleasanton, California, United States

CL microscopy - the analysis of light emitted by a mineral or gemstone when excited by an electron source - is a very effective microanalysis tool used to understand our geological history and the quality of optoelectronic materials and, has proven critical in applications including mineral provenance for geochronology and metamorphic alteration studies [1] and thermobaromtery [2].

Many CL detectors only allow spatial information to be collected in the form of black and white images. However, the lack of spectral (wavelength) information available in this data, prevents the identification of trace elements, their valence and, structural position for quantitative analysis. Consequently, color and spectrum imaging approached (hyperspectral imaging) have become more common. It almost goes without saying that spectrum imaging offers significant benefits over other analysis techniques with all spatial and spectral information collected in a single data set (x, y, l-referred to as a spectrum image or hyperspectral data cube).

Traditionally, CL detectors captured spectrum images by collecting wavelength-resolved spectra on a point-by-point basis using a pixelated detector such as a CCD camera [3]. This approach captures spectrum images with high spectral resolution $(0.1-1 \mathrm{~nm}$ typically) however, due to the low signal levels, slow frame rates and (read out) noise of the detector, it was often necessary to compromise spatial sampling in order to capture data in a reasonable timeframe. We report an alternative approach to capturing spectrum image data cubes which enables higher spatial sampling with a trade-off in spectral resolution.

The new acquisition paradigm is performed by collecting a series of (aligned) wavelength-filtered maps using (ultra-fast) photomultiplier tube detectors to build the spectrum image data cube, this approach is termed wavelength-filtered spectrum imaging. For use cases where spectral resolution better than a few nanometers is not required (as is the case in many geological studies), this approach offers significant benefit in acquisition speed or increased spatial sampling.

In Figure 1, we compare two hyperspectral images of a polished zircon grain captured using the wavelength-filtered spectrum imaging and traditional wavelength-resolved modes using the Monarc detector (Gatan Inc); data sampling was set so that the total acquisition time was the same in both experiments. Comparison of the extracted spectra demonstrate that all significant spectral features could be resolved in just 42 wavelength channels. In this example, the new approach to CL spectrum imaging allowed for $>70 x$ higher spatial sampling, enabling the fine banding structure to be resolved. 

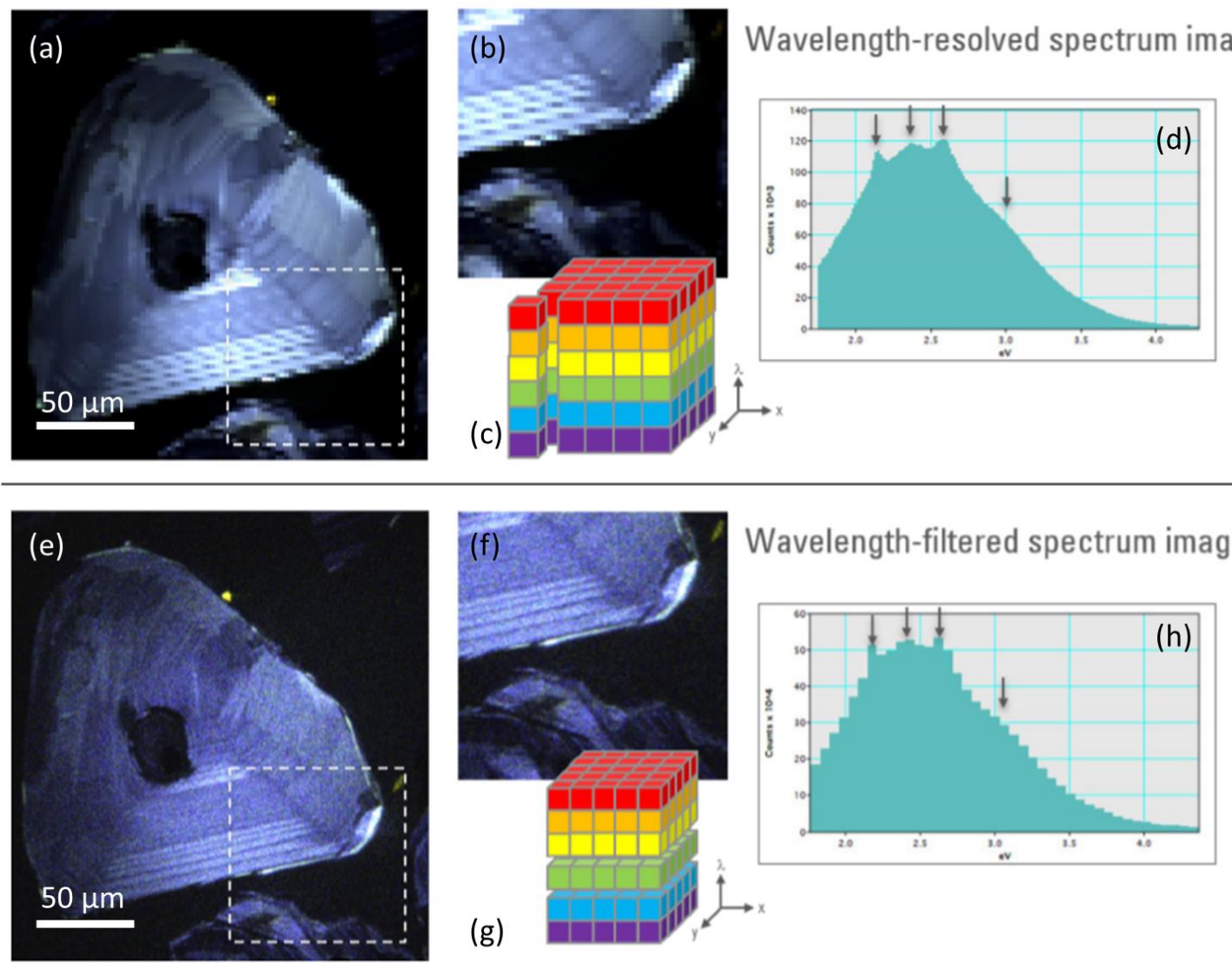

Wavelength-filtered spectrum imaging

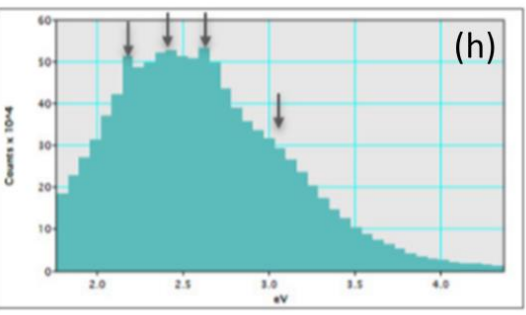

Figure 1. $(\mathrm{a}-\mathrm{d})$ Wavelength-resolved (WR-) and (e $-\mathrm{h})$ wavelength-filtered spectrum images (WF-SIs) of a polished zircon grain. True-color representations of the spectrum image data cubes are shown in (a), (b), (e) and (f) with the higher spatial sampling of the WF-SI approach enabling the fine banding to be resolved (f).

\section{References}

[1] T. Okumuraa et al., Quarternary Geochronology 3 (2008) p342.

[2] C MacRae et al., Microsc. and Micro. 16 (2010) p. 808.

[3] S Galloway, P Miller, P Thomas and R Harmon, Phys. Stat. Sol. C 0 (2003) p. 1028. 